\title{
Midpalatal implants for orthodontic anchorage
}

\author{
Abstracted from \\ Sandler J, Benson PE, Doyle P, et al. \\ Palatal implants are a good alternative to headgear: a randomized trial. \\ Am J Orthod Dentofacial Orthop 2008; 133: 51-67. \\ Address for correspondence: Jonathan Sandler, Chesterfield Royal Hospital, \\ Chesterfield, S44 5BL, UK. E-mail: JonSandler@aol.com
}

\section{Question: In patients requiring supplementing anchorage during orthodontic treatment are midpalatal implants as effective as headgear?}

\section{Design Randomised controlled trial}

Intervention Patients with various malocclusions in which any forward movement of the molars would prevent achievement of an ideal Class I canine relationship were randomly allocated to either the headgear or the implant group. Randomisation was carried out by using a block design and computer-generated random numbers. The allocations were concealed in consecutively numbered, sealed envelopes. Outcome measure The outcomes were; surgical and orthodontic success rates of the implants, the number of visits, the length of treatment time and the success of treatment as judged by the peer assessment rating (PAR) score reductions and the patients' attitudes to implant placement.

Results The surgical success rate of the implants was $75 \%$ and the orthodontic success rate was more than $90 \%$. Both implants and headgear proved to be effective methods of reinforcing anchorage. The total number of visits was greater in the implant group, but the overall treatment times were almost identical. There were no statistically significant differences between the 2 groups in PAR scores either at the start or the end of treatment, and the percentages of PAR score reductions were almost identical. The patients had no problems accepting midpalatal implants as a method of reinforcing anchorage.

Conclusions The use of palatal implants to reinforce anchorage was as effective as extraoral anchorage with headgear.

\section{Commentary}

The purpose of this trial was to assess the effectiveness of midpalatal implants compared to headgear as supplemental anchorage for orthodontic treatment in patients with different types of malocclusions. The authors selected a randomised clinical trial which was a suitable design to compare the effectiveness of treatment interventions. The number of participants was based upon a power calculation which also accounted for dropout rate. This criterion was appropriate considering the length of follow up needed. Patients were excluded based upon poor oral hygiene and their unwillingness to accept the treatment modality. Additionally, clefts and craniofacial syndromes were excluded to reduce the possibility of bias. Computer-generated randomisation and allocation concealment was appropriately used and provided two groups of similar baseline characteristics. Outcomes were assessed similarly in both groups using dental and cephalometric analysis. Information on patient centred outcomes was also evaluated. No statistically significant differences were reported for treatment length (excluding osseointegration time) and PAR scores.

Levels of discomfort during implant placement and after surgery were acceptable for most of the patients, but no patients' outcomes for acceptability and discomfort were evaluated in the headgear group. The authors were surprised by the compliance levels within the headgear group which they attributed to a Hawthorne Effect and higher levels of information associated with trial participation.

Out of 24 implant patients there were six surgical failures. Four of the six successfully integrated during a second attempt. This $74 \%$ success rate is lower than that reported in recent systematic reviews and may reflect the degree of experience required for proper placement.

Mini implant or temporary anchorage devices (TADS) are being used worldwide for orthodontic anchorage. They are rapidly gaining acceptance as they require very minimal patient compliance as compared to the headgear. Interestingly, findings in this study revealed that compliance was not a relevant issue.

The success of mini implants depends upon several factors among which are the surgical technique, the size and diameter of the implants and the forces applied to the implants. Several studies and systematic reviews ${ }^{1,2}$ have examined these factors and their significance, however this is still a relatively new method and specialists are gradually accepting and becoming more acquainted with this relatively new technique.

\section{Practice point}

- Mini midpalatal implants for orthodontic anchorage seem to perform as well as anchorage with headgear (when patient compliance is not a concern for the clinician).

Silvia Spivakovsky and Analia V Keenan NYU College of Dentistry, New York, USA.

1. Chen Y, Kyung HM, Zhao WT, Yu WJ. Critical factors for the success of orthodontic mini-implants: a systematic review. Am J Orthod Dentofacial Orthop 2009; 135: 284-291.

2. Reynders R, Ronchi L, Bipat S. Mini-implants in orthodontics: a systematic review of the literature. Am J Orthod Dentofacial Orthop 2009; 135: 564.e1-19. 